\title{
Classification of Diabetic Retinopathy using Stacked Autoencoder-Based Deep Neural Network
}

\author{
1Yasir Eltigani Ali Mustafa, \& ${ }^{2}$ Bashir Hassan Ismail \\ ${ }^{1}$ Department of Information Systems, Ahmed Bin Mohamed Military College, Doha, Qatar. \\ ${ }^{2}$ Breaking Barriers, United Kingdom. \\ **Corresponding Author: yasir@abmmc.edu.qa
}

Received: 05.01 .2020 , Revised: 08.02.2020, Accepted: 17.03.2020, Published: 30.03 .2020

DOI:

10.53409/mnaa.jcsit1102

\begin{abstract}
Diagnosis of diabetic retinopathy (DR) via images of colour fundus requires experienced clinicians to determine the presence and importance of a large number of small characteristics. This work proposes and named Adapted Stacked AutoEncoder (ASAE-DNN) a novel deep learning framework for diabetic retinopathy (DR), three hidden layers have been used to extract features and classify them then use a Softmax classification. The models proposed are checked on Messidor's data set, including 800 training images and 150 test images. Exactness, accuracy, time, recall and calculation are assessed for the outcomes of the proposed models. The results of these studies show that the model ASAE-DNN was $97 \%$ accurate.
\end{abstract}

Keywords: Adapted Stacked AutoEncoder, diabetic retinopathy, deep learning and Deep Neural Network.

\section{INTRODUCTION}

ne of the most advanced organs, the human eye is retinal, pupil, iris, lens and optical nerve. Appears as an effective screening method for early detection for eye disease automated retinal image analysis. Retinopathy (DR) and glaucoma, uncontrolled diabetes, can lead to blindness. Diabetes mellitus is elevated blood glucose chronic condition due to either insulin shortage or resistance to insulin [1,2].

The worldwide diabetes prevalence was found at around 425 million in 2017 and is expected to increase to about 630 million in 2045 [3]. In India, about a fifth to a third (57 million) of all people with Diabetes Mellitus (DM) will have retinopathy by 2025. Amongst them, about 5.7 million diabetes sufferers will suffer from extreme retinopathy and will need a laser or surgical operation to maintain vision [4].

Convolutional Neural Networks (CNNs), a deep learning branch, has an outstanding performance of applications such as medical imaging and analysis of images. This work provides the two-stage DR detection method based on the model with retinal images with these motives. At first, the image data of MESSIDOR is used as an input. In the model
ASAE-DNN, the DNN-based system is used with DR classification Adapted Stacked Automobile Encoders (ASAE-DNN).

The rest of the paper is written like this. Section 2 deals with the classification of DR related plays. Section 3 describes the methods proposed for defining the DR classification with ASAE-DNN. Section 4 discusses the experimental findings. Section 5 includes the conclusion and prospective work.

\section{II.RELATED WORK}

A good computer-aided clinical decision support system was developed to identify retinal images using the neural network and introduced [5]. The literature proposed different methods for the identification of DR. With and without moderate retinopathy, and Bayesian ANN has been educated to distinguish between healthy and diabetic eyes [6]. The proposed methodology to modelling based on $m$-medios [7] was extended and combined with a Gaussian model of a mixture in an ensemble to constructing a hybrid level classification to enhance grade accuracy.

A new automated screening scheme is proposed to support diabetic retinopathy [8] which involves the automatic selection, 
screening and classification of colour fundus pictures of diabetic retinopathy that might help diagnose and control diabetic retinopathy.

A novel automated identification with the use of fluid image processing techniques for diabetic retinopathy and maculopatherapy with eye-fundus images [9]. The Machine Learning Bagging Ensemble Classifier (ML-BEC) is designed to classify retinal features for DR disease diagnosis and early detection using computer teaching and ensembling methods [10]. A comprehensive deep learning algorithm based on data was designed and evaluated as a new DR detection diagnostic tool [11].

\section{PROPOSED METHODOLOGY}

Figure 1 displays the overall design of the Adapted Auto-Encoder stack for Diabetic retinopathy with a Deep neural network. The network uses an image data MESSIDOR as input on the first level and generates a low-level fixedlength function vector from the input. No function engineering is necessary, and the extractor immediately learns in the secret layer, contrary to several traditional DR methods. The ASAE-DNN extracts the data collection with ASAE and classifies the dataset with softmax row. Then, three hidden layers cross the lowlevel feature vector, and softmax has been used to measure the diabetic retinopathy classification result. During the following supervised training step, the final classification layer (output layer) is applied to practice the final Diabetic Retinopathy predictive models.

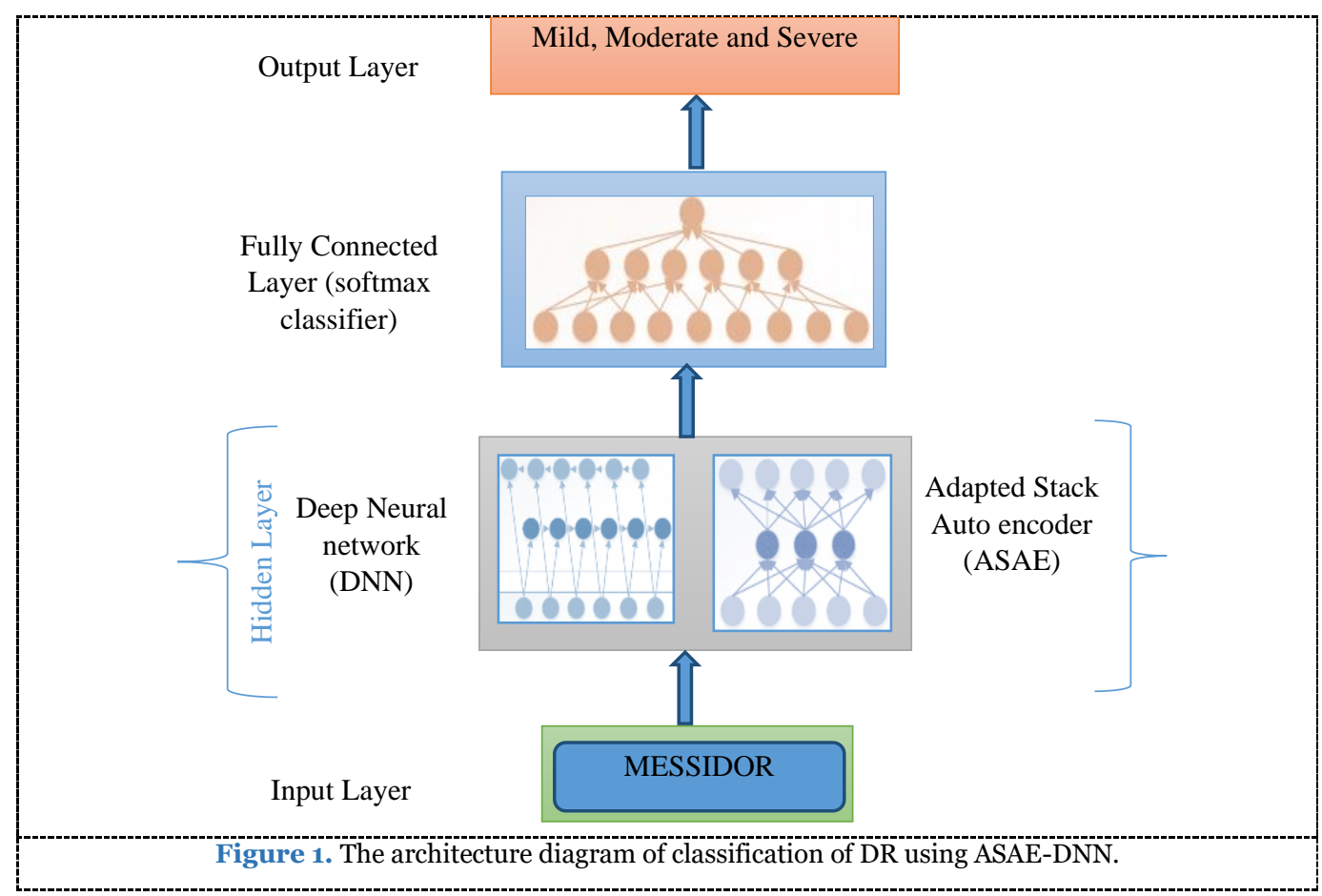

\subsection{Input MESSIDOR dataset}

In this work, to identify DR, a benchmark MESSIDOR dataset was used [12]. This data had approximately 1200 colour fundus images with proper annotation. The images in the dataset were categorized into four categories. Image grading was performed on the existence of microaneurysms, and hemorrhages were allocated to the images. The image without any symptoms indicates healthy retina. The image which has some microaneurysms represents stage 1 (Mild) whereas the image with some microaneurysms as well as hemorrhages denotes stage 2 (Moderate). The images that indicate more microaneurysms, as well as hemorrhages that are placed under stage 3 (Severe).

\subsection{Diabetic Retinopathy classification Using ASAE-DNN model}

Motivated by the fascinating features of deep networks, this work proposes an examination of the entire classification problem using DNN-based structures using adapted stacked autoencoders (ASAE-DNN). The DNN diabetes dataset classifier is built with an adapted autoencoder stacked with three hidden layers of extraction, and the last hidden layer to the classification process is attached to the softmax layer. The output layer gives the 
probabilities for a specific record in the diabetic and non-diabetic groups.

Consider an ASAE network with three layers, let $I=$ $1,2, \ldots n$ signifies the raw input of MESSIDOR images also $I^{\prime}$ is the reconstruction of the image as well as $w^{(l, 1)}, w^{\prime(l, 1)}, b^{(l, 1)}, b^{\prime(l, 1)}$ that are the weights and bias terms for the $l$ th layer encoder and decoder, individually, $l=1,2,3$. Primarily, the SAE maps the input image keen on a hidden design hid as signified in equation (1)

$$
\begin{aligned}
& \sum_{i=1}^{n} \sum_{l=1}^{3} h i d_{i}^{(l)}=\sum_{i=1}^{n} \sum_{l=1}^{3} f\left(I_{i}^{(l)}\right) \\
& f\left(I_{i}^{\prime(l)}\right)=s_{f}\left(w^{(l, 1)} I_{i}^{I^{(l)}}+b^{(l, 1)}\right)
\end{aligned}
$$

Then the latent representation hid $(l)$ mapped back into a reconstruction $\mathbb{R}$ from a corrupted version $I^{\prime}$ as in Eq.(2)

$$
\begin{gathered}
\mathbb{R}=g\left(\sum_{i=1}^{n} \sum_{l=1}^{3} h i d_{i}^{(l)}\right) \\
g\left(I^{l}\right)=s_{g}\left(w^{\prime(l, 1)} \sum_{i=1}^{n} \sum_{l=1}^{3} h i d_{i}^{(l)}+b^{(l, 1)}\right)
\end{gathered}
$$

where $s_{f}$ and $s_{g}$ stand nonlinear activation function. The cost function for SAE well-defined through the reconstruction error $\mathbb{R}\left(I^{\prime}, \mathbb{R}\right)$ between original input $I^{\prime}$ and reconstruction $\mathbb{R}$ as in equation (3):

$$
=\sum_{l=1,2,3} \mathbb{R}\left(I^{\prime(l)}, g\left(\sum_{i=1}^{n} \sum_{l=1}^{3} f\left({I^{\prime}}_{i}^{(l)}\right)\right)\right)
$$

Where $\mathbb{R}\left(I^{\prime}, \mathbb{R}\right)=\left\|I^{\prime}-\mathbb{R}\right\|^{2}$. Moreover, sparsity constraints term $\mathbb{T}$ is added into hidden units of ASAE to improved learning features, and an additional penalty term is introduced into the objective function in equation (4). Then and there the new cost function of ASAE can be rewritten as in equation (4):

$$
\begin{aligned}
& J_{M S A E}(\theta)=J_{A S A E}+\mathbb{W} \sum_{j=1 \text { to } m} K L(\mathbb{T} \\
& \| \mathbb{A}_{j} \text { ) }
\end{aligned}
$$

The additional penalty terms Kullback-Leibler divergence (KL) in equation (5) is well-defined by the cross-entropy in the middle of $\mathbb{T}$ and $\mathbb{A}_{j}$.

$$
\begin{aligned}
K L\left(\mathbb{T} \| \mathbb{A}_{j}\right)= & \sum_{j=1}^{m} \mathbb{T} \log \frac{\mathbb{T}}{\mathbb{A}_{j}} \\
& +(1-\mathbb{T}) \log \frac{1-\mathbb{T}}{1-\mathbb{A}_{j}} \\
\mathbb{A}_{j}= & \frac{1}{k} \sum_{i=1}^{k} h_{i}
\end{aligned}
$$

Here, $\mathbb{T}$ designate the target sparsity level, $\mathbb{A}_{j}$ denotes the average activation rate for the $j$ th unit, $m$ remains the number of hidden units, $\mathbb{W}$ stands the weight for penalty terms. The sparsity dictions code for each layer represents the input data in the quest for a collection of over-complete base vectors to achieve a better image function. The intrinsic feature of an image is learned by a three-layer scattered coding process, as the sparse representation at pixel level created the feature. Cascading with the softmax classifier is possible to construct a deep network classifier through stacking an autoencoder. Stacked auto encoder may have two or more layers of auto encoders. Let $\left\{I_{1}, \ldots, I_{n}\right\}$ variables be $\left\{Y_{1}, \ldots, Y_{n}\right\}$ input vector given as input to DNN besides the corresponding output class, here $n=3$. The training method aims to change the DNN parameters to know the input vectors to identify the appropriate output with greater accuracy if the input vectors for the training are assumed. The instructional classification procedure ASAE-DNN is as described in the following:

1. The first autoencoder layer is typically achieved with the original input vector $\left\{I_{1}, \ldots, I_{n}\right\}$ such as the same vector as the destination. This layer tries to recreate the input by removing the $\left\{f_{(1,1)}, \ldots, f_{(1, i)}\right\}$ functions.

2. In addition to yielding the output vector of the first autoencoder layer $\left\{o_{(1,1)}, \ldots, o_{(1, i)}\right\}$ as an input vector, the second autoencoder layer is equipped by enchanting the output vector as input vector and generates an output vector $\left\{o_{(2,1)}, \ldots, o_{(2, j)}\right\}$. The second autoencoder layer attempts to recreate the input $o_{(1, i)} ; i=1,2, \ldots, C$.

3 . The third autoencoder layer is equipped by deciding to take the output vector as the input vector and generates the output vector of the second autoencoder layer $\left\{c_{(2,1)}, \ldots, c_{(2, j)}\right\}$ as the input vector and generates the output vector $\left\{c_{(3,1)}, \ldots, c_{(3, k)}\right\}$. The third layer of autoencoders attempts to recreate the o $o_{(2, j)} ; j=1,2, \ldots, R$ data.

4. The stacked autoencoder is cascaded via SVM with the softmax classifier layer [13] which will increase the accuracy of DNN classification. This layer is trained through taking output of the third autoencoder layer, $c_{(3, k)} ; k=1,2, \ldots, U$ as the input vector and the original class variables $\left\{Y_{(1)}, \ldots, Y_{(N)}\right\}$ as the query sequence from the training data.

5. Eventually, backpropagation would be used which called fine is tuning, improving the efficiency of the DNN classification. The network is made redundant in some kind of controlled manner with the training data. Once the first hyperplane classification is defined by a back propagation algorithm, the training process is terminated whether it is local or global optimum. The SVM classification hyperplanes are suitable globally by using a comprehensive risk minimisation framework.

\section{EXPERIMENTAL RESULTS AND DISCUSSION}

The performance of the proposed ASAEDNN is evaluated in this section, and the performance results are compared with existing Neural Network [4], ML-BEC [9] and deep learning 
[10] schemes. The performance measurement is done in terms of precision, f-measurement, recall and accuracy.

Precision: It reflects the proportion of positive samples correctly classified as expected in equation (5):

$$
\text { Precision }=\frac{T P}{F P+T P}
$$

Recall: The recall of a classifier reflects the positive samples properly assigned to the total number of positive samples and is calculated as in equation (6):

$$
\text { Recall }=\frac{T P}{T P+F N}
$$

F-measure: this is also referred to as F 1-score, and as in equation (7) is the harmonic mean of precision and recall:

$$
F-\text { measure }=\frac{2 *(\text { Recall } * \text { Precision })}{(\text { Recall }+ \text { Precision })}
$$

Accuracy: This is one of the most frequently used performance classification measures and is defined as a ratio between the correctly classified samples and the total number of samples as in equation (8):

$$
\text { Accuracy }=\frac{T P+T N}{T P+T N+F P+F N}
$$

Where true positive (TP) samples are properly classified as no DR, false positive (FP) samples are incorrectly classified as DR, True negative (TN) samples are properly classified as DR, and false negatives (FN) are incorrectly classified as DR.

\subsection{Precision Rate comparison}

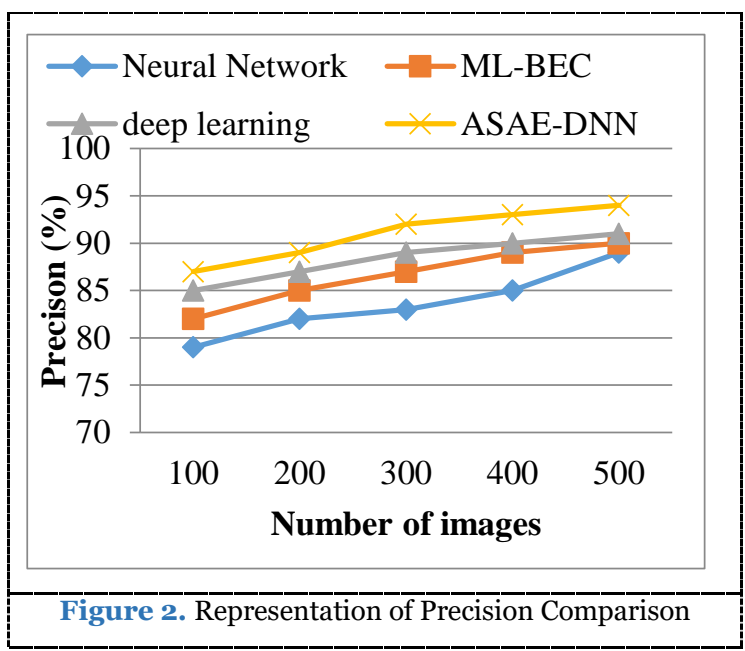

From the above Figure 2, the graph shows how accurate the number of images in the specified datasets is compared. These methods are implemented as Neural Network, ML-BEC and deep learning and ASAE-DNN. When the number of records increases according to the precision value, from this graph, it is learned that the proposed ASAE-DNN offers $94 \%$ higher precision than previous methods that yield better results in the classification of $\mathrm{CR}$ due to adapted stacked autoencoder.The numerical results of Precision Comparison is shown in Table 1.

Table 1. The numerical results of Precision Comparison

\begin{tabular}{ccccc}
\hline $\begin{array}{c}\text { No.of } \\
\text { images }\end{array}$ & $\begin{array}{c}\text { Neural } \\
\text { Network }\end{array}$ & $\begin{array}{c}\text { ML- } \\
\text { BEC }\end{array}$ & $\begin{array}{c}\text { deep } \\
\text { learning }\end{array}$ & $\begin{array}{c}\text { ASAE- } \\
\text { DNN }\end{array}$ \\
\hline 100 & 79 & 82 & 85 & 87 \\
\hline 200 & 82 & 85 & 87 & 89 \\
\hline 300 & 83 & 87 & 89 & 92 \\
\hline 400 & 85 & 89 & 90 & 93 \\
\hline 500 & 89 & 90 & 91 & 94 \\
\hline
\end{tabular}

4.2. Recall comparison

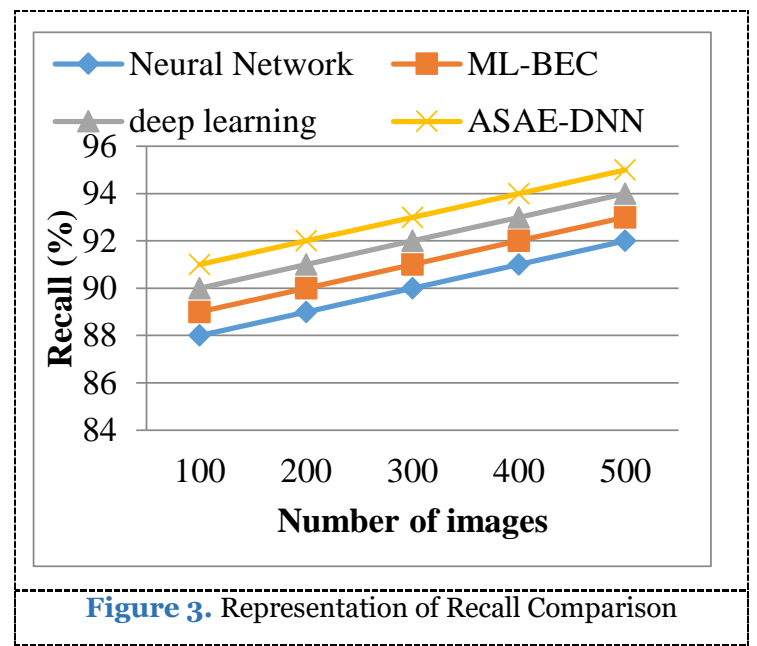

From the above Figure 3, the graph illustrates the recall relation for the number of images in the listed datasets. These methods are implemented as Neural Network, ML-BEC and deep learning. Increasing the number of images often increases the correct value for the recall. Through this graph, it is discovered that the current ASAE-DNN offers recall 95\% higher than previous methods. The explanation for this is that the ASAE-DNN extracts the features directly, which will enhance the detection and classification of DR. The numerical results of Recall Comparison is shown in Table 2.

Table 2. The numerical results of Recall Comparison

\begin{tabular}{ccccc}
\hline $\begin{array}{c}\text { No.of } \\
\text { image } \\
\mathbf{s}\end{array}$ & $\begin{array}{c}\text { Neural } \\
\text { Networ } \\
\mathbf{k}\end{array}$ & $\begin{array}{c}\text { ML- } \\
\text { BE } \\
\mathbf{C}\end{array}$ & $\begin{array}{c}\text { deep } \\
\text { learnin } \\
\mathbf{g}\end{array}$ & $\begin{array}{c}\text { ASAE } \\
\text {-DNN }\end{array}$ \\
\hline 100 & 88 & 89 & 90 & 91 \\
\hline 200 & 89 & 90 & 91 & 92 \\
\hline 300 & 90 & 91 & 92 & 93 \\
\hline 400 & 91 & 92 & 93 & 94 \\
\hline 500 & 92 & 93 & 94 & 95 \\
\hline
\end{tabular}




\subsection{F-measure Rate comparison}

From the above Figure 4, the graph explains the f-measure relation for the number of images in the given datasets. These methods are implemented as Neural Network, ML-BEC and deep learning.

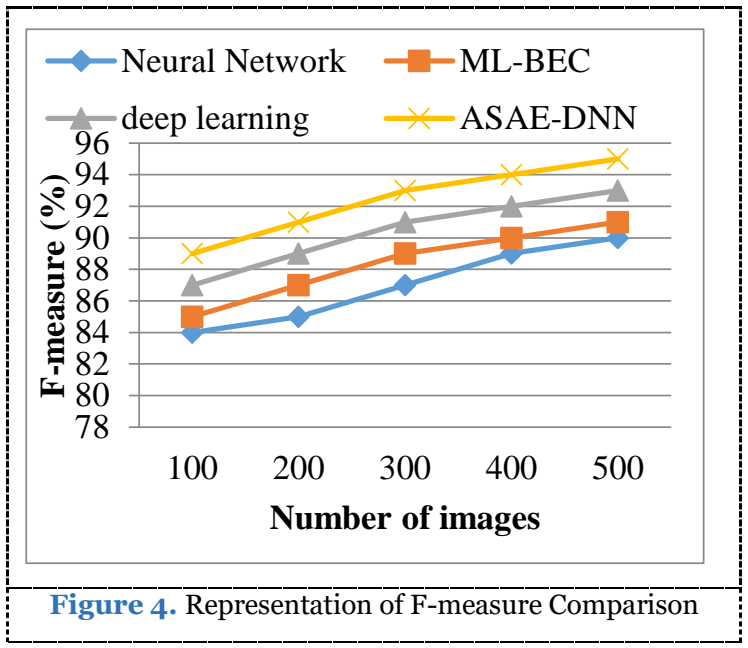

When the number of data is increased, and the fmeasure value is increased accordingly. From this graph it is learned that the proposed ASAE-DNN offers 95\% higher f-measurement than previous methods. Therefore the proposed ASAE-DNN algorithm is stronger than the current algorithms in terms of better performance of classifying DR. The numerical results of F-measure Comparison is shown in Table 3.

Table 3. The numerical results of F-measure Comparison

\begin{tabular}{ccccc}
\hline $\begin{array}{c}\text { No.of } \\
\text { images }\end{array}$ & $\begin{array}{c}\text { Neural } \\
\text { Network }\end{array}$ & $\begin{array}{c}\text { ML- } \\
\text { BEC }\end{array}$ & $\begin{array}{c}\text { deep } \\
\text { learning }\end{array}$ & $\begin{array}{c}\text { ASAE- } \\
\text { DNN }\end{array}$ \\
\hline 100 & 84 & 85 & 87 & 89 \\
\hline 200 & 85 & 87 & 89 & 91 \\
\hline 300 & 87 & 89 & 91 & 93 \\
\hline 400 & 89 & 90 & 92 & 94 \\
\hline 500 & 90 & 91 & 93 & 95 \\
\hline
\end{tabular}

\subsection{Accuracy comparison}

From the above Figure 5, the diagram illustrates the processing time relation for the number of images in the specified datasets. These methods are implemented as Neural Network, MLBEC and deep learning and ASAE-DNN. From this graph, it is known that the proposed ASAE-DNN algorithm is higher than the existing algorithms with a high precision rate of $97 \%$ in terms of better template matching results. This is due to the automatic extraction of the function in the ASAEDNN algorithm, which increases the DR classification results. The numerical results of Accuracy Comparison is shown in Table 4.

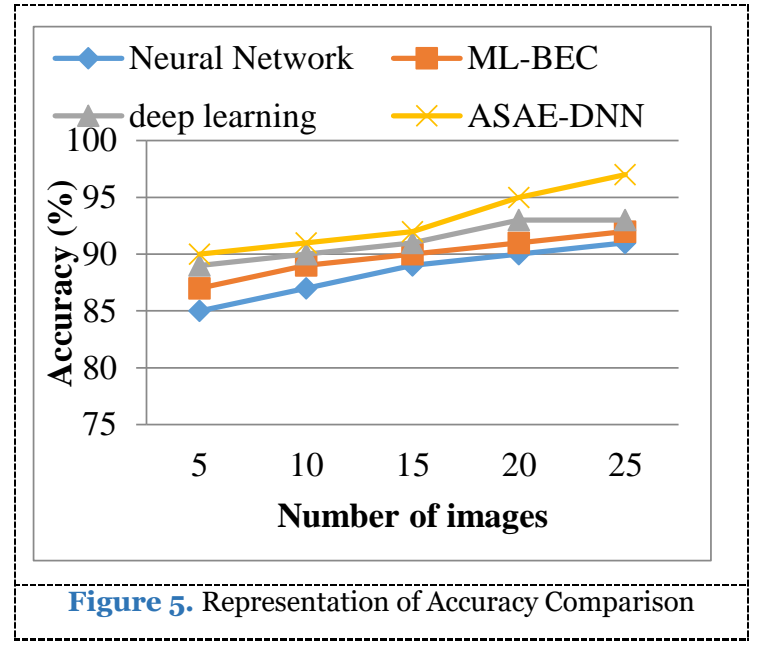

Table 4. The numerical results of Accuracy Comparison

\begin{tabular}{ccccc}
\hline $\begin{array}{c}\text { No.of } \\
\text { images }\end{array}$ & $\begin{array}{c}\text { Neural } \\
\text { Network }\end{array}$ & $\begin{array}{c}\text { ML- } \\
\text { BEC }\end{array}$ & $\begin{array}{c}\text { deep } \\
\text { learning }\end{array}$ & $\begin{array}{c}\text { ASAE- } \\
\text { DNN }\end{array}$ \\
\hline 5 & 85 & 87 & 89 & 90 \\
\hline 10 & 87 & 89 & 90 & 91 \\
\hline 15 & 89 & 90 & 91 & 92 \\
\hline 20 & 90 & 91 & 93 & 95 \\
\hline 25 & 91 & 92 & 93 & 97 \\
\hline
\end{tabular}

\section{CONCLUSION AND FUTURE WORK}

This study proposes that ASAE-DNN algorithms use stacked auto-encoders, and that three hidden layers be used for extraction of features accompanied by a softmax classifier classification for DR classification with a high accuracy rate of $97 \%$. It provides a reliable solution for DR detection within a large-scale data set, as well as the results achieved indicate the good efficiency of today's computeraided model in providing efficient, low-cost and objective DR diagnostics without any need for clinicians to manually examine and grade images. In the future, it may also be important to investigate different types of common patient metadata, such as genetic factors, patient history, duration of diabetes, hemoglobin $\mathrm{A} 1 \mathrm{C}$ value, and other clinical data that may influence a patients at risk of retinopathy. Introducing this information to the classification model could give informative correlations to following DR risk factors from outside strictly imaging information, increase thermal diagnostic accuracy.

ETHICS APPROVAL AND CONSENT TO PARTICIPATE

Not applicable.

HUMAN AND ANIMAL RIGHTS 
No animals/humans were used for studies that are basis of this research.

\section{CONSENT FOR PUBLICATION \\ Not applicable.}

AVAILABILITY OF DATA AND MATERIALS

The authors confirm that the data supporting the findings of this research are available within the article.

\section{FUNDING}

None.

\section{CONFLICT OF INTEREST}

The authors declare no conflict of interest, financial or otherwise.

\section{ACKNOWLEDGEMENTS}

The authors would like to thank their present employer for providing support while carrying out this research work.

\section{REFERENCES}

[1]. DeFronzo RA, Ferrannini E, Zimmet P, Alberti KG. International Textbook of Diabetes Mellitus. 4th ed.., Vol. 2. Oxford UK: Wiley-Blackwell; 2015.

[2]. International Diabetes Federation. IDF Diabetes Atlas. 8th ed.. International Diabetes Federation; 2017. Available from: http://www.diabetesatlas.org/. [Last accessed on 2019 May 01].

[3]. Balasubramaniyan N, Ganesh Kumar S, Ramesh Babu K, Subitha L. Awareness and practices on eye effects among people with diabetes in rural Tamil Nadu, India. Afr Health Sci 2016;16:210-7.

[4]. Kumar SJ, Madheswaran M. An improved medical decision support system to identify the diabetic retinopathy using fundus images. Journal of medical systems. 2012 Dec 1;36(6):3573-81

[5]. Mookiah MR, Acharya UR, Martis RJ, Chua CK, Lim CM, Ng EY, Laude A. Evolutionary algorithm based classifier parameter tuning for automatic diabetic retinopathy grading: A hybrid feature extraction approach. Knowledgebased systems. 2013 Feb 1;39:9-22.

[6]. Somfai GM, Tátrai E, Laurik L, Varga B, Ölvedy V, Jiang H Wang J, Smiddy WE, Somogyi A, DeBuc DC. Automated classifiers for early detection and diagnosis of retinopathy in diabetic eyes. BMC bioinformatics. 2014 Dec 1;15(1):106.

[7]. Akram MU, Khalid S, Tariq A, Khan SA, Azam F. Detection and classification of retinal lesions for grading of diabetic retinopathy. Computers in biology and medicine. $2014 \mathrm{Feb}$ 1;45:161-71.

[8]. Rahim SS, Jayne C, Palade V, Shuttleworth J. Automatic detection of microaneurysms in colour fundus images for diabetic retinopathy screening. Neural computing and applications. $2016 \mathrm{Jul}$ 1;27(5):1149-64.

[9]. Rahim SS, Palade V, Shuttleworth J, Jayne C. Automatic screening and classification of diabetic retinopathy and maculopathy using fuzzy image processing. Brain informatics. 2016 Dec 1;3(4):249-67.
[10]. Somasundaram SK, Alli P. A machine learning ensemble classifier for early prediction of diabetic retinopathy. Journal of Medical Systems. 2017 Dec 1;41(12):201.

[11]. Gargeya R, Leng T. Automated identification of diabetic retinopathy using deep learning. Ophthalmology. $2017 \mathrm{Jul}$ 1;124(7):962-9.

[12]. MESSIDOR dataset: Available at: http://www.adcis.net/en/third-party/messidor/.

[13]. W. Liu, Y. Wen, Yu Z, et al. Large-margin softmax loss for convolutional neural networks, 507-516 (2016) 